DEPARTMENT OF THE INTERIOR

JPIVE

NEW WEX...
RECONNAISSANCE FOR RADIOACTIVITY

IN THE GOLD HILL MINING AREA, BOULDER COUNTY, COLORADO, PART 1

By

R. H. Campbell

This report is preliminary and has not been edited or reviewed for conformity with U. S. Geological Survey standards and nomenclature.

February 1955

United States Geological Survey

Washington, D. C.

Prepared by the Geological Survey for the UNITED STATES ATOMIC ENERGY COMMISSION Technical Information Service, Oak Ridge, Tennessee 
This report concerms work done on behalf of the Division of Raw Materials of the U. S. Atomic Energy Commiseion.

The Atomic Energy Commission makes no representation or warranty as to the accuracy or wefulness of the information or statements contained in this report, or that the we of any information, apparatus, method or process disclosed in this report may not infringe privately-owned rights. The Commission assumes no llabllity with respect to the use of, or for damages resulting from the we of, any Information, apparatus, method or process disclosed in this report.

This report has been reproduced directly from the best avallable copy.

Printed in USA, Price 25 cents. Available from the Office of Technical Services, Department of Conmerce, Washington 25, D. C. 


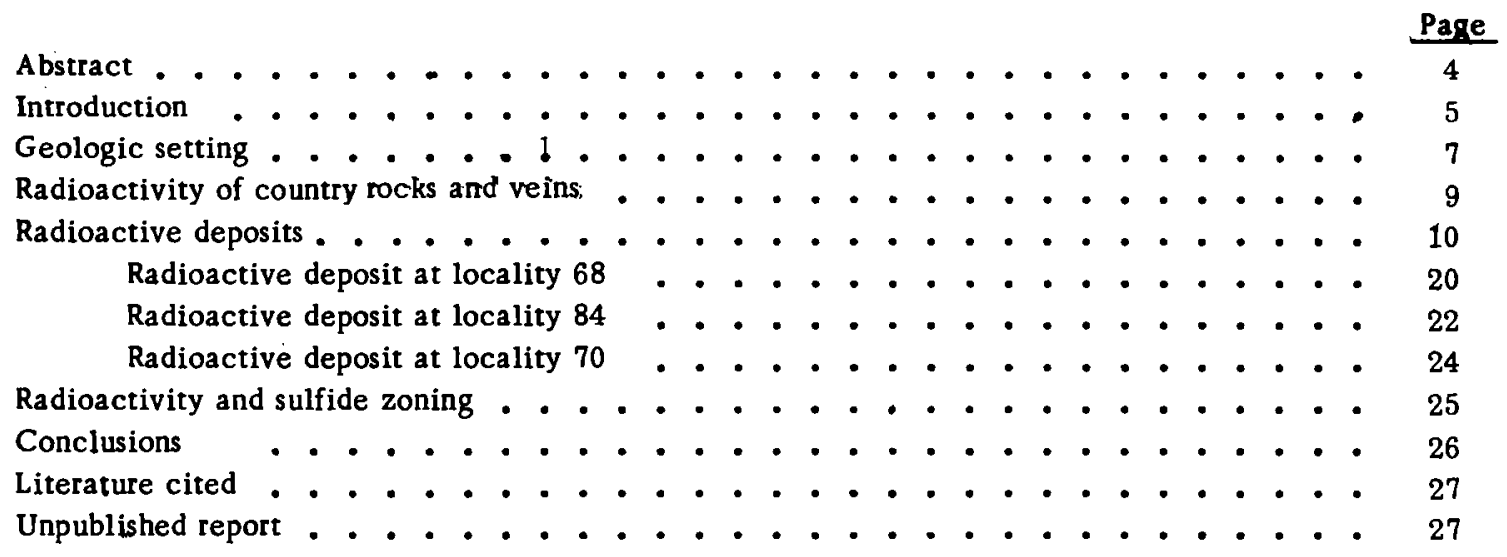

ILLUSTRATIONG

Figure 1. Index maps showing location of Gold Hill mining area, Boulder County, Colorado $\frac{\text { Page }}{6}$

2. Generalized geologic map showing localities examined for radioactivity in the Gold Hill mining area, Boulder County, Colorado . . . . . . . . . In envelope

3. Geologic sketch of workings at locality 68 , Gold Hill mining area, showing places sampled . . . . . . . . . . . . . . 21

4. Geologic map and sections of workings at locality 84, Gold Hill mining area, showing known extent of radioactive deposit . . . . . . . . . . .

T A BLE

Table 1. Tabulated descriptions of localities examined for radioactivity in the Gold Hill mining area, Boulder County, Colorado . . . . . . . . . . . 12 


\title{
RECONNAISSANCE FOR RADIOACTIVITY IN THE GOLD HILL \\ MINING AREA, BOULDER COUNTY, COLORADO
}

By R. H. Campbe1l

\begin{abstract}
Severail radioactive deposits were found as a result of reconnaissance in the Gold Hill mining area, Boulder County, Colo. The ore deposits of the area have been worked chiefly for gold. All ore shipped has come from fissure veins, most of which are gold telluride veins. There are, however, some important sulfide veins which show a vague zonal distribution of pyritic gold ores and silverlead ores. The results of this reconnaissance suggest a possible relationship of the radioactive deposits to this indistinct sulfide zoning; however, the zoning is so obscure that its practical application to prospecting for uranium is of doubtful value at the present time.

Pitchblende, torbernite, metatorbernite, and schroeckingerite have been identified in specimens from the area; however, no uranium minerals have yet been identified from most of the radioactive deposits, and the uraniferous material present is probably in disseminated small particles. Although selected samples from several localities assay 0.10 percent uranium or more, the known deposits are small and probably are not of immediate economic importance.
\end{abstract}




\section{INTRODUCTION}

The Gold Hill mining area is in the central part of Boulder County, Colo. , about 5 miles northwest of Boulder, and includes an area of about 12 square miles (fig. 1). It lies near the northern end of the Front Range mineral belt just south of the Jamestown district. The largest settlement in the area is Gold Hill, but several other small mining camps are also included. All are accessible the year around from Boulder. The ore deposits of the area have been worked chiefly for gold. All ore shipped has come from fissure veins, most of which are gold-telluride veins; there are, however, some important pyritic gold veins and a few important silver-lead veins.

Several deposits of radioactive material were found as a result of one month's field reconnaissance for radioactivity in the Gold Hill area. The field work was done by the writer with the valuable assistance of Max Schafer during April 1953. More than 90 localities were examined for radioactivity. and those showing abnormally high radioactivity were sampled. In 84 mines and prospects a total of about 45,000 feet of underground workings were traversed with a scintillation counter. It is estimated that not more than 50 percent of the accessible mine workings in the area have been examined for radioactivity. Twenty-two dumps, several prospect pits, and about 2.500 feet of surface stopes, vein outcrops, and breccia reef outcrops were examined for radioactivity.

Radioactive material had been reported from several mines in the Gold Hill area, including pitchblende at the Black Cloud mine (King, ms. in preparation). A sketch map compiled in 1952 by A. R. McReel of the U. S. Geological Survey indicated a zonal distribution of sulfide ores, and it was noted that the position of the Black Cloud mine was roughly intermediate between a group of pyritic gold veins and an area containing silver-lead veins. Because this pattern is similar to that noted for the pitchblende deposits at Central City, Colo. (Leonard, 1952), the Gold Hill area was chosen for study by the Geological Survey's project on "relation of uranium to hypogene mineral zoning" under the general

supervision of B. F. Leonard. The study was made to assess the importance of mineral zoning as a guide to uraniferous deposits in this area. 


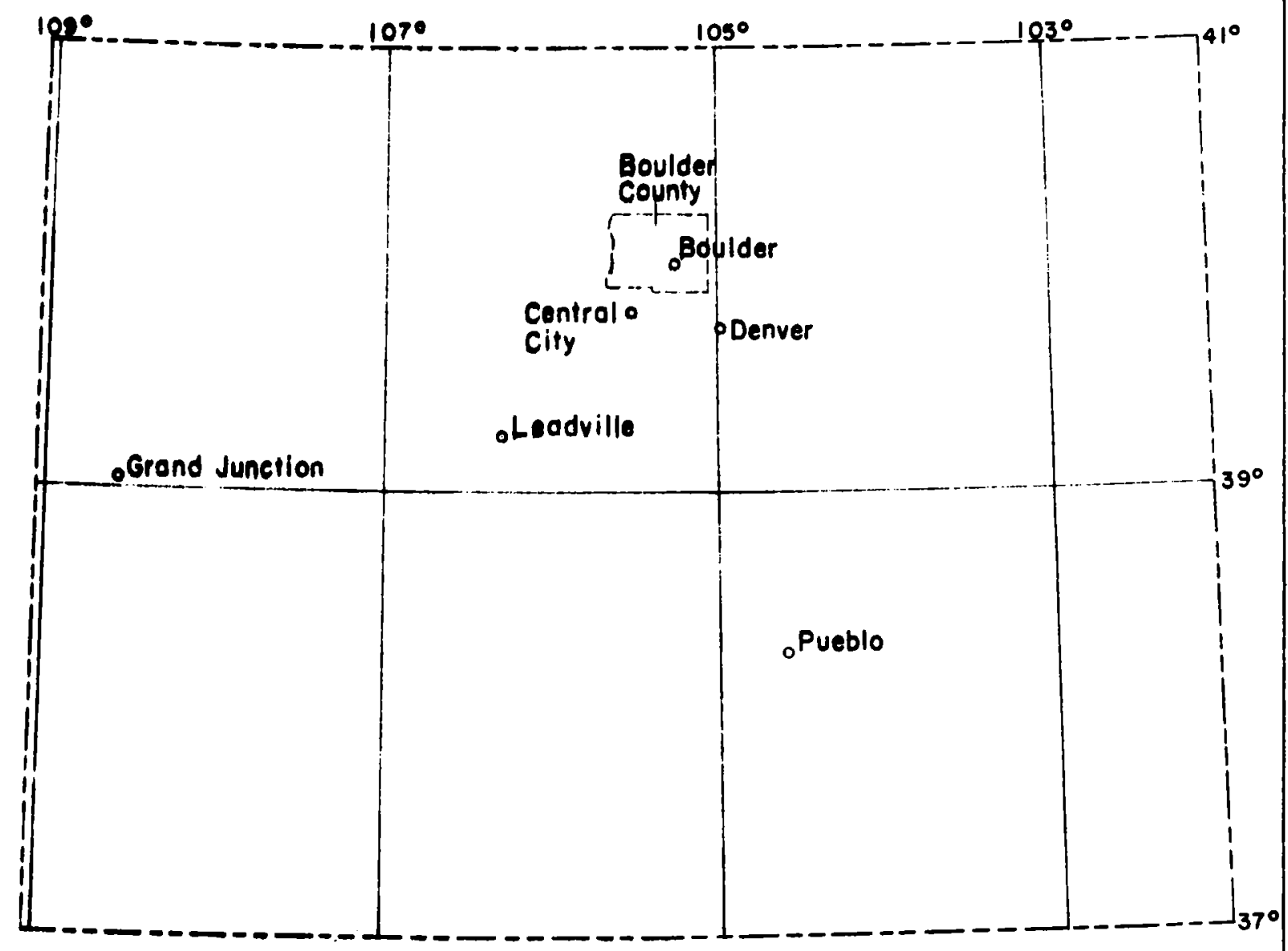

MAP OF COLORADO SHOWING LOCATION OF BOULDER COUNTY

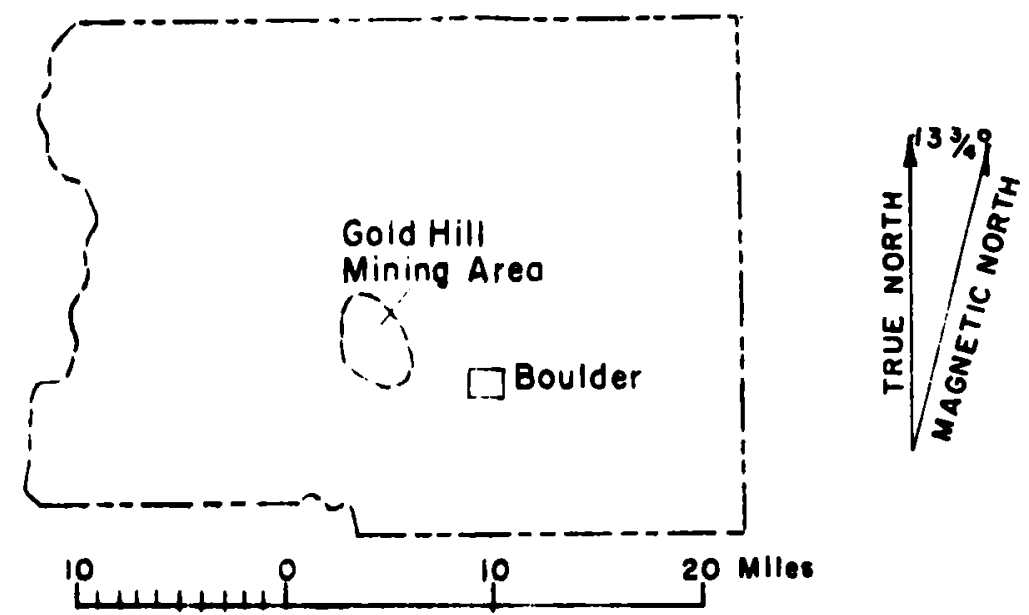

MAP OF BOULDER COUNTY SHOWING LOCATION OF GOLD HILL MINING AREA

FIGURE I. - INDEX MAPS SHOWING LOCATION OF GOLD HILL MINING AREA,BOULDER COUNTY, COLORADO 
The geology of the Gold Hill mining area has been mapped in detail $(1 / 12,000)$ by E. N. Goddard (1940). Topographic maps covering the area are the U. S. Geological Survey Boulder quadrangle $(1 / 62,500)$ and the Geological Survey Gold Hill mining area $(1 / 12,000)$.

The writer wishes to express his appreciation to the mining men of the area and of Boulder for their assistance and cooperation; and to his colleagues of the U. S. Geological Survey for much stimulating discussion and helpful advice. The work was done by the U. S. Geological Survey on behalf of the Division of Raw Materials of the U. S. Atomic Energy Commission.

\section{GEOLOGIC SETTING}

Lovering (1932), Goddard (1940), and Lovering and Goddard (1950) have described the geology of the district, and the following brief description has been abstracted from these reports.

Precambrian rocks of the area include: the Boulder Creek granite which underlies nearly all of the district; schists of the Idaho Springs formation on the northern and western edges of the area which interfinger and wrap around the large granite body; and dikes, lenses, and irregular masses of gneissic granite aplite, quartz diorite. Silver Plume granite, and pegmatite and aplite. Dike rocks of Laramide age include diabase, quartz monzonite, alaskite, bostonite, and biotite monzonite and latite. On figure 2 all of the smaller units have been omitted, with the exception of the bostonite dikes which commonly show high radioactivity in the area.

Well developed northwest-trending breccia reef faults of Laramide age are present in the area. The reefs have considerable variation in width, intensity of shearing, and degree of silicification. They are classified, on the basis of strike and dip, into four sets. The Hoosier and Maxwell reefs are the most persistent and prominent in the area and belong to a set that trends $\mathrm{N}$. $25^{\circ}$ to $50^{\circ} \mathrm{W}$. with nearly vertical dips. The Poorman, Blue, and Bull $O^{\prime}$ the Woods reefs are included in another prominent but less persistent set trending $\mathrm{N}$. $70^{\circ}$ to $80^{\circ} \mathrm{W}$. with nearly vertical dips. A third set, including the Fortune reef, trends N. $60^{\circ}$ to $75^{\circ} \mathrm{W}$. and dips $30^{\circ}$ to $45^{\circ} \mathrm{NE}$. A fourth set includes small faults striking N. $5^{\circ}$ to $300 \mathrm{E}$. and dipping steeply northwest; this group has been largely obscured by later mineralization. 
Although commonly lacking important ore minerals themselves, the breccia reefs are believed to have had an important influence on the distribution of the ore deposits of the area. Most of them probably served as deep channels for the circulation of ore-forming solutions, but a few may have had a damming effect, blocking circulation in certain areas and directions.

Gold is the chief metal produced from mines of the Gold Hill area, but in most places minor amounts of silver are associated with the gold. Silver-has been the most important product of a few of the mines and some lead has been produced with it. The most important ore minerals in the area are the gold tellurides, although numerous veins locally carry abundant free gold. Very small amounts of galena and sphalerite are associated with the ore, and fine-grained pyrite is disseminated throughout the telluride veins, Pyrite and chalcopyrite are the most abundant ore minerals in the pyritic gold veins, but free gold is locally abundant. Gold seems to be associated with both chalcopyrite and pyrite. Small amounts of sphalerite, galena, and gray copper are commonly associated with the pyritic gold ore. The silver-lead ores contain the same minerals as the pyritic gold ores but in different amounts: tennantite (argentiferous gray copper) and galena are the chief ore minerals, but small amounts of sphalerite. chalcopyrite, and pyrite are present. Tungsten has been produced from ferberite ore in the Logan mine (fig. 2).

All ore shipped has come from fissure veins which are abundant throughout most of the district. The veins are fairly persistent; most of the productive veins are more than half a mile long, and a few are more than a mile long. Commonly, their width is between 1 and 5 feet, but local zones of shearing or fracturing are 10 to 30 feet wide and in some places even wider. Many veins have been followed to depths of 300 to 600 feet, and a few mines are considerably deeper.

Nearly all the veins strike northeast and dip steeply, but a few strike northwest and a few others, such as the King vein, are nearly horizontal. Goddard (1940) distinguished 2 main sets of northeast-striking veins. One set of north-northeast trend appears to be earlier. These fissures have been filled with the gold-telluride type of vein material and comprise most of the productive veins of the area. Another set, trending eastnortheast, includes fissures which commonly contain pyritic gold or lead-silver ores. A group of eastnortheast-trending veins occurs east of Emancipation Hill where they appear to be earlier than the northtnortheast-trending set. 


\section{RADIOACTIVITY OF COUNTRY ROCKS AND VEINS}

The average radioactivity on the surface over Boulder Creek granite and schists of the Idaho Springs formation is $0.02 \mathrm{mr} / \mathrm{hr}$ (milliroentgens per hour) in a usual range of 0.015 to $0.021 \mathrm{mr} / \mathrm{hr}$, with slightly higher radioactivity (up to $0.03 \mathrm{mr} / \mathrm{hr}$ ) locally. The variation between 0.015 and $0.021 \mathrm{mr} / \mathrm{hr}$ is probably mostly a result of variations in conditions governing cosmic-ray activity, as variations of this magnitude occurred at the same place over a period of several days and even within a few hours. The radioactivity of the pegmatites is commonly about $0.05 \mathrm{mr} / \mathrm{hr}$ and generally ranges from 0.04 to $0.08 \mathrm{mr} / \mathrm{hr}$; however, much higher readings are found locally. The bostonite dikes commonly have a radioactivity of $0.25 \mathrm{mr} / \mathrm{hr}$ or higher. Underground the radioactivity of the country rock, as measured in barren crosscuts, ranges from 0.02 to $0.04 \mathrm{mr} / \mathrm{hr}$ and is consistently very near the average reading of $0.03 \mathrm{mr} / \mathrm{hr}$.

Radioactive "fall out" resulting from atomic weapons tests in Nevada caused abnormally high radioactivity on the surface on several occasions. On days when measurements at previously visited surface localities of country rock indicated "fall our" had occurred, radioactivity measurements were reliable only in underground workings and were not taken on the surface.

Nearly all the veins examined are, over their entire exposures, more radioactive than the adjacent country rocks. The a verage radioactivity of individual veins is generally between 0.03 and $0.09 \mathrm{mr} / \mathrm{hr}$. and many average 0.05 and $0.06 \mathrm{mr} / \mathrm{hr}$. In addition, the dumps examined commonly show the highest radibactivity at places where vein material is most abundant on the surface of the dump. The higher radioactivity, therefore, appears to be characteristic of vein-filling material and associated altered and mineralized wall rock. This feature is observed consistently throughout the district regardless of zonal position, ore type, and proximity to breccia reefs and is found on veins far from known occurrences of uranium minerals as well as those near such occurrences. 
In some veins, radioactivity appeared to increase in direct proportion to the amount of vein material present. That is, radioactivity increased in or near stopes and visible swells on the vein. This relationship. however, is not consistent throughout the district, as many local "highs" appear to have no relationship to the thickness of the veins in which they occur. Some veins show an increased radioactivity at splits and intersections; however, this relationship also is not consistent throughout the district.

\section{RADIOACTIVE DEPOSITS}

All of the abnormally high radioactivity found in the area is in the veins or in altered country rock adjacent to the veins, except for the bostonite dikes and a few pegmatite bodies. The genetic relation of uraniferous material to the veins, however, is obscured, as all but one of the occurrences of uranium minerals may be secondpry, and the uraniumı may not be in the same position relative to the vein as that in which it was originally deposited. In most of the radioactive deposits no uranium minerals have as yet been identified, and the uraniferous material present is probably in disseminated small particles. In the few mines where uranium minerals have been observed those identified are pitchblende, torbernite, metatorbernite, and schroeckingerite.

Radioactive deposits had been reported from two mines in the area, the Black Cloud and the Snowbound, prior to this investigation (King, ms. in preparation). A sample of gold quartz vein material from the Snowbound mine and a sample from the Black Cloud mine containing quartz, pyrite, galena, sphalerite, and sooty pitchblende assayed, respectively 0.006 and 0.17 percent $U$. The Black Cloud was visited subsequently by the writer, J. W. Adams, and F. Stugard, Jr. . on September 28, 1953, at which time small clusters of crystals of a fluorescent radioactive mineral were observed as a post-mine deposit on the ribs of a drift. A sample of this mineral was identified by X-ray methods as schroeckingerite by W. F. Outerbridge of the U. S. Geological Survey, Denver Laboratory. 
Table 1 gives a brief description of the localities examined, the maximum radioactivity found. and analytical data for those sampled. Except where otherwise noted, the samples taken were from the most radioactive parts of anomalous areas and are intended to represent the maximum grade of uranium-bearing material present.

In many of the samples (table 1 ) the eU/U ratios are greater or less than one. Several samples analyzed by J. N. Rosholt, Jr. . U. S. Geological Survey .Deirter Laboratmy, indicate that'this is caused by a disequilibrium between uranium and its disintegration products. No appreciable thorium was found in any of the samples. Most of the samples showing disequilibrium have aU/E iratios greater than one. This could result from either: (1) differential leaching of uranium leaving a residue of radioactive daughter products, or (2) by selective deposition of radioactive decay products of uranium (probably chiefly radium) in the absence of equilibrium amounts of the parent uranium. It is believed that differential leaching of uranium by ground water is the more probable. In the few samples having $\mathrm{eU} / \mathrm{U}$ ratios less than one,the disequilibrium is probably the result of recent deposition of uranium which has not been in place long enough to establish equilibrium with its disintegration products.

Three radioactive deposits, localities 68,84, and 70 , are described in the following sections in more detail than that given on table 1 . All three contain visible uranium minerals in veins, but each is somewhat different from the others in mineralogy and mode of occurrence. The deposit at locality 68 contains sooty pitchblende (?) and pyrite in a narrow vein. Locality 70 contained the only "hard" pitchblende found. The deposit at locality 84 contains torbernite, metatorbernite, and sooty pitchblende(?) chiefly in altered granite in the hanging wall of a vein. 
Table 1. --Tabulated descriptions of locallties examined for radioactivity in the Gsld Hill mining area, Boulder County, Colorado.

\begin{tabular}{|c|c|c|c|c|c|c|c|c|c|c|c|c|c|}
\hline \multirow[b]{2}{*}{$\begin{array}{l}\text { Lecality } \\
\text { number } \underline{1} /\end{array}$} & \multirow[b]{2}{*}{$\begin{array}{l}\text { Coordi- } \\
\text { àates } 1 /\end{array}$} & \multirow[b]{2}{*}{ Name } & \multirow{2}{*}{$\begin{array}{l}\text { Type of } \\
\text { workings } \\
\text { examined }\end{array}$} & \multirow{2}{*}{$\begin{array}{l}\text { Length of } \\
\text { workings } \\
\text { traversed } \\
\text { (feet) } 2 /\end{array}$} & \multirow{2}{*}{$\begin{array}{l}\text { Maximum } \\
\text { radio- } \\
\text { activity } \\
(\mathrm{mr} / \mathrm{hr})\end{array}$} & \multicolumn{7}{|c|}{ Analytical data } & \multirow[b]{2}{*}{ Remarks $\underline{3}$ / } \\
\hline & & & & & & $\begin{array}{l}\mathrm{eU} \\
\text { (percent) }\end{array}$ & $\underset{\text { (percent) }}{U}$ & $\begin{array}{c}\text { Au } \\
\text { (oz/ton) }\end{array}$ & $\begin{array}{c}\mathrm{Ag} \\
(\mathrm{oz} / \mathrm{ton})\end{array}$ & $\begin{array}{c}\mathrm{Cu} \\
\text { (percent) }\end{array}$ & $\begin{array}{c}\mathrm{Pb} \\
\text { (percent) }\end{array}$ & $\frac{\mathrm{Zn}}{\text { (percent) }}$ & \\
\hline 1 & $C-5$ & Unknown & Crosscut adit & 100 & 0.06 & -- & -- & -- & $\cdots$ & -- & $\therefore$ & -- & Pegmatite at portal. \\
\hline 2 & $C-5$ & do. & do. & 10 & .05 & -- & -- & -- & -- & -- & -- & -- & Pegmatîte at portal. \\
\hline 3 & $C-5$ & do. & $\begin{array}{l}\text { Locked drift } \\
\text { adit; and dump } \\
\text { at shaft }\end{array}$ & 0 & .08 & $-\cdot$ & -- & -- & $\cdots$ & -. & -- & -- & Vein at portal of locked adit. \\
\hline 4 & B-3 & do. & $\begin{array}{l}\text { Drift adit. } \\
\text { drifts, stopes }\end{array}$ & 2,500 & .09 & $-\cdot$ & -- & -- & -- & -- & -- & $-\cdot$ & \\
\hline 5 & $8-3$ & Ingram. & Drift adit & 1.000 & 1.50 & -- & -- & -- & -- & -- & -- & -- & \\
\hline 6 & $C-4$ & King. & Upper drift adit & 1.500 & .25 & -- & -- & -- & - & $\cdots$ & -- & -- & Highly fractured. altered Boulder Creek granite. \\
\hline 7 & $C-1$ & Unknown & Crosscut & 2.500 & .08 & 0.004 & 0.001 & Tr. & $\mathbf{3 . 2}$ & -- & -- & -- & Ten-pound sample of vein material from stope. \\
\hline 8 & $c-1$ & do. & Dump & 0 & .04 & - & -- & - & $\cdots$ & -- & -- & $-\cdot$ & \\
\hline 9 & $C-1$ & do. & Drift adit & 30. & .08 & -- & -- & - & $-\cdot$ & -- & -- & - & Silicified and limonite-stained cross ifracture. \\
\hline 10 & $C-1$ & do. & $\begin{array}{l}\text { Two short } \\
\text { drift adits }\end{array}$ & 50 & .12 & -- & - & -- & $\cdots$ & $\cdots$ & $-\cdots$ & -- & \\
\hline 11 & D-5 & Poorman & $\begin{array}{l}\text { Crosscut adit, } \\
\text { drifts, stopes }\end{array}$ & 1.700 & .10 & $\cdots$ & -- & - & $\cdots$ & -- & -- & -- & \\
\hline 12 & $D-5$ & Unknown & $\begin{array}{l}\text { Crosscut adit, } \\
\text { drifts, stopes }\end{array}$ & 500 & .20 & .005 & .001 & 1.08 & 1.20 & .02 & 0.01 & 0.002 & $\begin{array}{l}\text { Vein with quartz and finely disseminated pyrite, } \\
\text { chalcopyrite (?), and galena (?). }\end{array}$ \\
\hline
\end{tabular}

1/ As shown on figure 2

2/ Footage recorded only for underground workings, surface stopes, and vein and breccia reef outcrops. 3/ Descriptions of types of material associated with the maximum radioactivity listed. Except where otherwise noted the radioactivity is associated with vein material showing no megascopically
visible ore minerals. 
Table 1. - Tabalated descriptions of localties examined for radioactivity in the Gold Hill mining area Boulder County Colosado. --Continued

\begin{tabular}{|c|c|c|c|c|c|c|c|c|c|c|c|c|c|}
\hline \multirow[b]{2}{*}{$\begin{array}{l}\text { Locality } \\
\text { number } 1 /\end{array}$} & \multirow[b]{2}{*}{$\begin{array}{l}\text { Coordi- } \\
\text { nates } 1 /\end{array}$} & \multirow[b]{2}{*}{ Name } & \multirow{2}{*}{$\begin{array}{l}\text { Type of } \\
\text { workings } \\
\text { examined }\end{array}$} & \multirow{2}{*}{$\begin{array}{l}\text { Length of } \\
\text { workings } \\
\text { traversed } \\
\text { (feet) } 2 /\end{array}$} & \multirow{2}{*}{$\begin{array}{l}\text { Maximum } \\
\text { radio- } \\
\text { activity } \\
(\mathrm{mr} / \mathrm{hr})\end{array}$} & \multicolumn{7}{|c|}{ Analyzical data } & \multirow[b]{2}{*}{ Remarks $\underline{\underline{\theta}} /$} \\
\hline & & & & & & $\begin{array}{l}\mathrm{eU} \\
\text { (percent) }\end{array}$ & $\begin{array}{l}\mathrm{U} \\
\text { (percent) }\end{array}$ & $\begin{array}{l}\mathrm{Au} \\
(\mathrm{ioz} / \mathrm{ton})\end{array}$ & $\begin{array}{l}\mathrm{Ag} \\
(\mathrm{oz} / \mathrm{ton})\end{array}$ & $\begin{array}{c}\mathrm{Cu} \\
\text { (percent) }\end{array}$ & $\begin{array}{c}\mathrm{Pb} \\
\text { (percent) }\end{array}$ & $\begin{array}{c}\mathbf{Z n} \\
\text { (percent) }\end{array}$ & \\
\hline 13 & D-5 & Poorman & $\begin{array}{l}\text { Surface stopes. } \\
\text { vein and breccia } \\
\text { reef outcrops, } \\
\text { prospect pits }\end{array}$ & 2.000 & .12 & -- & -- & $\cdots$ & -- & -- & $\cdots$ & $\cdots$ & Outcrop of vein meterial. \\
\hline 14 & $C-1$ & Unknown & $\begin{array}{l}\text { Vein outcrops, } \\
\text { surface stopes, } \\
\text { prospect pits }\end{array}$ & 100 & .04 & -- & -- & -- & -- & -- & - & -- & \\
\hline 15 & C-1 & Unknown & Drift & 60 & 0.06 & -- & -- & -- & -- & $\cdots$ & -- & $-\cdot$ & \\
\hline 10 & $C-1$ & 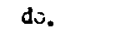 & do. & 75 & .03 & -- & -- & -- & $-\cdot$ & $\cdots$ & -- & -- & \\
\hline 17 & $C-1$ & do. & do. & 400 & .06 & -- & -- & -- & -- & -- & - & -- & \\
\hline 18 & $C-1$ & do. & do. & 1,100 & .12 & -- & -- & -- & -- & -- & - & -- & $\bar{\omega}$ \\
\hline 19 & B-2 & do. & do. & 150 & .13 & 0.007 & 0.002 & Tr. & 0.10 & -- & -- & -- & \\
\hline 20 & B-1 & do. & do. & 100 & .05 & .002 & -. & 0.00 & .00 & $\angle 0.01$ & $\angle 0,01$ & 0.01 & Thin fracture along main shear zone. \\
\hline 21 & B-2 & do. & do. & 100 & .03 & -- & -- & -- & -- & -- & -- & -- & \\
\hline \multirow[t]{2}{*}{22} & B-2 & do. & do. & 1.200 & .10 & .012 & .004 & .0 & .00 & 0.05 & $\angle 0.01$ & 0.12 & $\begin{array}{l}\text { Sample } 22 \text { from cross fracture about } \\
1,200 \text { ft. from portal. }\end{array}$ \\
\hline & & & & & .08 & .003 & -- & Tr. & .28 & .06 & $<01$ & .07 & Sample 22A. Bulk sample of vein material \\
\hline 23 & $\mathrm{C}-2$ & do. & Crosscut adit" & 20 & .03 & $\cdots$ & -- & -- & -- & -- & -- & -- & \\
\hline 24 & B-3 & do. & $\begin{array}{l}\text { Crosscut adit, } \\
\text { drifts; stopes }\end{array}$ & 800 & .15 & .025 & .014 & .0 & .0 & .05 & $\angle 01$ & .08 & \\
\hline 25 & $B-3$ & do. & Drift adit & 750 & .10 & -- & -- & -- & -- & -- & -- & -- & Cross fracture in main shear zone. \\
\hline 26 & $\mathrm{C}-3$ & do. & do. & 75 & .08 & -. & -- & -- & -- & -- & -- & -- & \\
\hline 27 & $C-3$ & Logan & $\begin{array}{l}\text { Drift adit. } \\
\text { drifts and stopes } \\
\text { on several veins }\end{array}$ & 4.000 & .12 & -- & $-\cdot$ & -- & -- & -- & -- & -- & Yein cut nearest:portal. \\
\hline
\end{tabular}


Table 1. --Tabulated descrptions of locolities examined for radioactivity in the Gold Hill mining area. Boulder County. Colorado--Continued

\begin{tabular}{|c|c|c|c|c|c|c|c|c|c|c|c|c|c|}
\hline \multirow[b]{2}{*}{$\begin{array}{l}\text { Locality } \\
\text { number } \underline{1} /\end{array}$} & \multirow[b]{2}{*}{$\begin{array}{l}\text { Coordi- } \\
\text { nates } 1 \text { / }\end{array}$} & \multirow[b]{2}{*}{ Name } & \multirow{2}{*}{$\begin{array}{l}\text { Type of } \\
\text { workings } \\
\text { examined }\end{array}$} & \multirow{2}{*}{$\begin{array}{l}\text { Length of } \\
\text { workings } \\
\text { traversed } \\
\text { (feet) } 2 /\end{array}$} & \multirow{2}{*}{$\begin{array}{l}\text { Maximum } \\
\text { radio- } \\
\text { activity } \\
(\mathrm{mr} / \mathrm{hr})\end{array}$} & \multicolumn{5}{|c|}{ Analytical data } & \multicolumn{2}{|r|}{$=$} & \multirow[b]{2}{*}{ Remarks 3 - } \\
\hline & & & & & & $\begin{array}{l}\text { eU } \\
\text { (percent) }\end{array}$ & $\begin{array}{l}\mathrm{U} \\
\text { (percent) }\end{array}$ & $\begin{array}{c}\mathrm{Au} \\
(\mathrm{oz} / \mathrm{ton})\end{array}$ & $\begin{array}{l}\mathrm{Ag} \\
\text { (oz/ton) (per }\end{array}$ & Cu & $\begin{array}{c}\mathrm{Pb} \\
\text { (percent) }\end{array}$ & $\frac{Z_{n}}{\text { (percent) }}$ & \\
\hline 28 & $C-3$ & $\begin{array}{l}\text { Grand } \\
\text { Republic }\end{array}$ & Open stopes & 300 & .06 & -- & -- & -- & - & - & $-\cdot$ & $\cdots$ & \\
\hline 29 & $C-5$ & Unknown & Crosscut adit & 100 & .08 & -- & -- & -- & $\cdots$ & -- & -- & -- & \\
\hline 30 & $C-4$ & do. & Drift adit & 100 & .05 & -- & -- & -- & -- & $\cdots$ & -- & $\cdots$ & \\
\hline 31 & $C-1$ & do. & do. & 100 & .04 & -- & -- & -- & -- & -- & -- &.- & \\
\hline 32 & $C-1$ & do. & do. & 100 & .06 & -- & -- & -- & $\cdots$ & $\cdots$ & $\cdots$ & -- & \\
\hline $33 \mathrm{U}$ & $C-4$ & do. & Crosscut adit & 100 & . 15 & -- & -- & -- & $-\cdot$ & $\cdots$ & $\cdots$ & -- & $\begin{array}{l}\text { Highly iractured, altered Bouider Creek } \\
\text { granite with thin quartz stringers. }\end{array}$ \\
\hline 332 & $C-4$ & do. & do. & 250 & .07 & $-\cdot$ & -- & -- & -- & -- & -- & $\cdots$ & Do. \\
\hline \multirow[t]{2}{*}{34} & $c-4$ & $\begin{array}{l}\text { King } \\
\text { tunnel }\end{array}$ & $\begin{array}{l}\text { Crosscut adit, } \\
\text { drifts, large stopes }\end{array}$ & 600 & 0.35 & 0.25 & 0.51 & \multicolumn{2}{|c|}{ Insufficient sample } & 0.07 & 0.02 & 0.10 & $\begin{array}{l}\text { Sample no. } 34-- \text { chip of material } \\
\text { coaring fracture in main stope. }\end{array}$ \\
\hline & & & & & .50 & .069 & .092 & Tr. & 0.08 & $<0.01$ & .02 & .09 & $\begin{array}{l}\text { Sample no. } 34 \text { a--chip from vein } \\
\text { about } 50 \mathrm{ft} \text {. from face of crosscut. }\end{array}$ \\
\hline 35 & $A-2$ & Unknown & Drift adit. & 550 & .40 & 1,24 & .96 & \multicolumn{2}{|c|}{ Insufficient sample } & $<01$ & $<01$ & .11 & Sample $35 A--$ Chip from vein at face, \\
\hline$\therefore \Leftrightarrow$ & & & & & .30 & .013 & .007 & Tr. & Tr. & $\ll 01$ & $<01$ & .12 & $\begin{array}{l}\text { Sample } 358 \text {-Bulk sample from vein } \\
\text { about } 60 \mathrm{ft} \text {. from portal. }\end{array}$ \\
\hline 36 & B-1 & do. & $\begin{array}{l}\text { Crosscut adit, } \\
\text { drifts, and stopes }\end{array}$ & 700 & .50 & .050 & .029 & Tr. & .14 & $<01$ & $<.01$ & .15 & $\begin{array}{l}\text { Chip from vein near face of northemmost } \\
\text { drift. }\end{array}$ \\
\hline 37 & B-1 & do. & Drîft adit & 150 & .09 & $-\cdot$ & $-\cdot$ & -- & -- & -- & -- & $\cdots$ & \\
\hline $38 \mathrm{U}$ & B-1 & do. & do. & 30 & .08 & -- & -- & -. & $\cdots$ & $=$ & -- & - & \\
\hline 38L & B-1 & do. & do. & 200 & .25 & -- & -. & -. & -- & -- & -- & -- & \\
\hline
\end{tabular}


Table 1. --Tabulated descriptions of localities examined for radioactivity in the Gold Hill mining area. Boulder County. Colorado--Continued.

\begin{tabular}{|c|c|c|c|c|c|c|c|c|c|c|c|c|c|}
\hline \multirow[b]{2}{*}{$\begin{array}{l}\text { Locality } \\
\text { number } 1 /\end{array}$} & \multirow[b]{2}{*}{$\begin{array}{l}\text { Coordi- } \\
\text { nates } 1 \text { - }\end{array}$} & \multirow[b]{2}{*}{ Name } & \multirow{2}{*}{$\begin{array}{l}\text { Type of } \\
\text { workings } \\
\text { examined }\end{array}$} & \multirow{2}{*}{$\begin{array}{c}\text { Length of } \\
\text { workings } \\
\text { traversed } \\
\text { (feet) } 2{ }^{\prime}\end{array}$} & \multirow{2}{*}{$\begin{array}{l}\text { Maximum } \\
\text { radio- } \\
\text { activity } \\
(\mathrm{mr} / \mathrm{hr})\end{array}$} & \multicolumn{5}{|c|}{ Analytical data } & & & \multirow[b]{2}{*}{ Remaiks 3 / } \\
\hline & & & & & & $\begin{array}{l}\text { eU } \\
\text { (percent) }\end{array}$ & $\frac{\mathrm{U}}{\text { (percent): }}$ & $\frac{\mathrm{Au}}{\mathrm{O} / \mathrm{z} / \mathrm{ton})}$ & $\begin{array}{c}\mathrm{Ag} \\
\text { (oz/ton) }\end{array}$ & $\begin{array}{c}\mathrm{Cu} \\
\text { (percent) }\end{array}$ & $\begin{array}{c}\mathbf{P b} \\
\text { (percent) }\end{array}$ & $\frac{\mathrm{Zn}_{\mathbf{n}}}{\text { (percent) }}$ & \\
\hline \multirow[t]{2}{*}{39} & $B-2$ & King & $\begin{array}{l}\text { Drift adit, } \\
\text { and dump } \\
\text { of shaft }\end{array}$ & 200 & .20 & -- & $-\cdot$ & $-\cdot$ & -- & -- & -- & $-\cdot$ & $\begin{array}{l}\text { Vein with sphalerite, pyrite, galena. } \\
\text { and chalcopyrite. }\end{array}$ \\
\hline & & & & & .06 & .002 & -- & .04 & 1.13 & .02 & 1.00 & .63 & $\begin{array}{l}\text { Sample no. } 39 \text {-grab sample of } \\
\text { radioactive vein matertal from dump. }\end{array}$ \\
\hline $39 a$ & B-2 & Unknown & Prospect pit & 0 & .04 & -- & -- & -- & -- & $\cdots$ & -- & -- & \\
\hline $39 b$ & B-2 & $\begin{array}{l}\text { Unknown } \\
\text { (on King } \\
\text { vein) }\end{array}$ & $\begin{array}{l}\text { Dump of caved } \\
\text { adit }\end{array}$ & 0 & .04 & -- & -- & -- & -- & -- & $\cdots$ & -- & $\begin{array}{l}\text { Vein with pyrite sphalerite, and } \\
\text { galena. }\end{array}$ \\
\hline $39 c$ & B-2 & do. & Dump & 0 & .06 & -- & -- & -- & -- & -- & -- & -- & \\
\hline 40 & B-2 & Unknown & Crosscut adit & 100 & .12 & -- & -- & -- & -- & - & -- & -- & En \\
\hline \multirow[t]{3}{*}{41} & B-2 & do. & Drift adit & 1.000 & .20 & .11 & .002 & .0 & .0 & .01 & $<01$ & .13 & $\begin{array}{l}\text { Sample } 41 \text { a-chip from fracture surface } \\
\text { on vein. }\end{array}$ \\
\hline & & & & & .40 & .17 & .21 & .0 & .0 & .01 & 601 & $\begin{array}{ll}.04 & \mathrm{~s} \\
& 3\end{array}$ & $\begin{array}{l}\text { Sample no. } 41 \mathrm{~b} \text {-chip of vein about } \\
30 \mathrm{ft} \text {, from face. }\end{array}$ \\
\hline & & & & & .15 & .004 & -- & Tr. & Tr. & $<01$ & « 01 & $\begin{array}{rr}.02 & 5 \\
& 7\end{array}$ & $\begin{array}{l}\text { Sample no. } 41 \mathrm{c}-\mathrm{chip} \text { of vein about } \\
70 \mathrm{ft} \text {. from portal. }\end{array}$ \\
\hline 42 & $C-4$ & do. & Crosscut adit & 20 & .04 & -- & $-\cdot$ & $-\cdot$ & $\cdots$ & -- & -- & -. & No vein exposed. \\
\hline 43 & $C-4$ & do. & Drift adit & 50 & $.05^{\circ}$ & -- & $-\cdot$ & -- & -- & $\cdots$ & -- & -- & \\
\hline 44 & $C-4$ & do. & do. & 50 & .07 & -- & -- & -. & -- & -- & -- & -- & \\
\hline 45 & $C-4$ & do. & do. & 50 & .08 & -- & $\cdots$ & -- & $\cdots$ & -- & -- & -- & \\
\hline 46 & B-3 & $\begin{array}{l}\text { Good Hope } \\
\text { tunnel }\end{array}$ & $\begin{array}{l}\text { Crosscut adit, } \\
\text { driftson } 3 \text { veins }\end{array}$ & 1,500 & 0.12 & -- & -- & -- & -- & -- & $\cdots$ & $\begin{array}{l}\mathrm{T} \\
\text { in }\end{array}$ & $\begin{array}{l}\text { Thin limonite coated fracture exposed } \\
\text { in crosscut. }\end{array}$ \\
\hline 47 & $B-4$ & Unknown & Drift adit & 70 & .04 & -- & -- & - & $\cdots$ & -- & -- & -- & \\
\hline 48 & B-4 & do. & do. & 150 & .20 & $-\cdot$ & -- & -- & -- & -- & -- & -. & \\
\hline
\end{tabular}


Table 1. --Tabulated descriptions of localities examined for tadioactivity in the Gold Hill mining area, Boulder County. Colorado--Colorado--Continued.

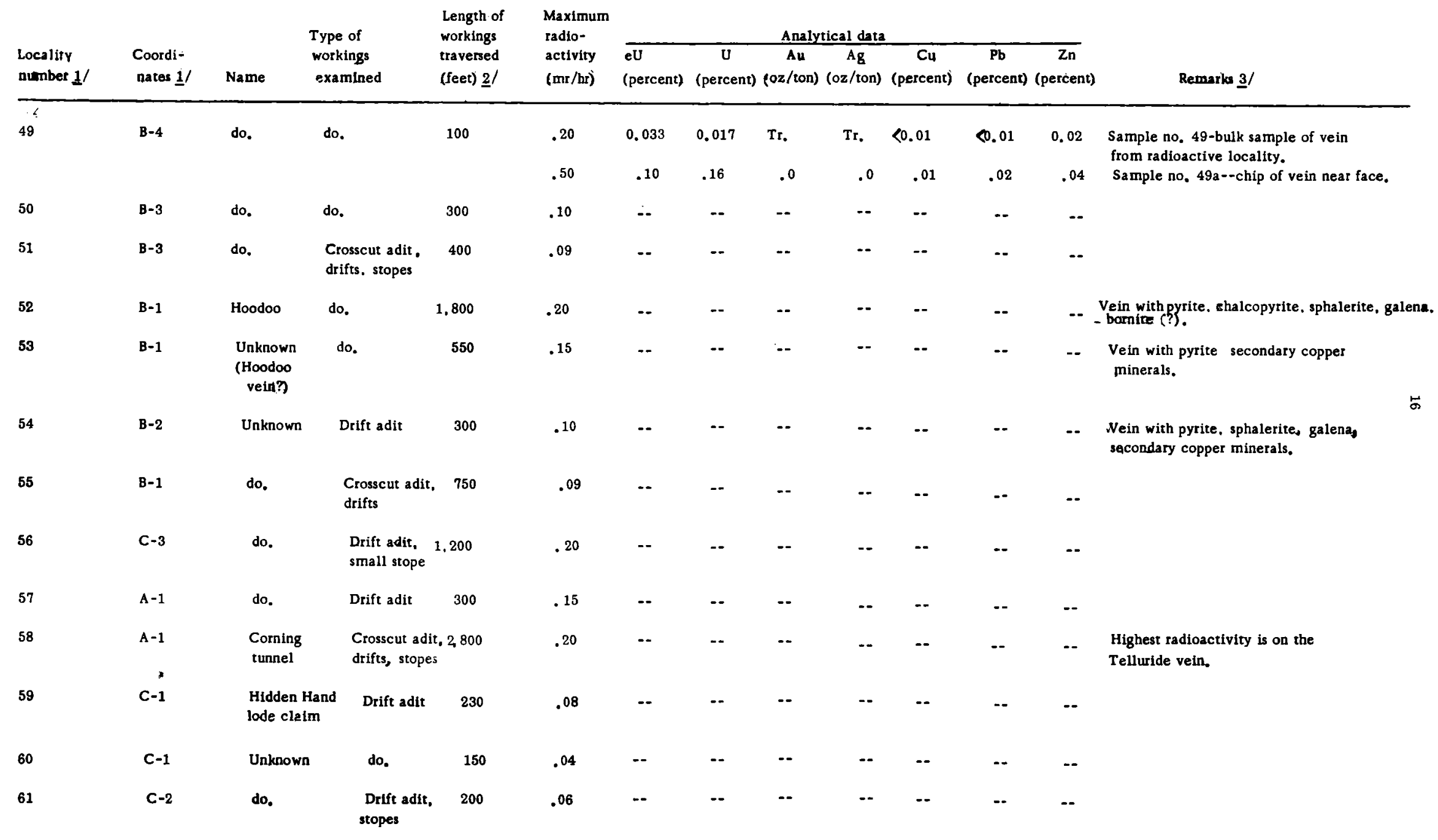


Table 1. --T abulated descriptions of localities examined for radioactivity in the Gold Hill mining area, Boulder County, Colorado--Continued.

\begin{tabular}{|c|c|c|c|c|c|c|c|c|c|c|c|c|c|}
\hline \multirow[b]{2}{*}{$\begin{array}{l}\text { Locality } \\
\text { number } 1 /\end{array}$} & \multirow[b]{2}{*}{$\begin{array}{l}\text { Coordi- } \\
\text { nates } \underline{1} /\end{array}$} & \multirow[b]{2}{*}{ Name } & \multirow{2}{*}{$\begin{array}{l}\text { Type of } \\
\text { workings } \\
\text { examined }\end{array}$} & \multirow{2}{*}{$\begin{array}{l}\text { Length of } \\
\text { workings } \\
\text { traversed } \\
\text { (feqt) } 2 /\end{array}$} & \multirow{2}{*}{$\begin{array}{l}\text { Maximum } \\
\text { radio- } \\
\text { activity } \\
\text { (mr/hr) }\end{array}$} & \multicolumn{7}{|c|}{ Analytical data } & \multirow[b]{2}{*}{ Remarks $\underline{3} /$} \\
\hline & & & & & & $\begin{array}{l}\text { eU } \\
\text { (percent) }\end{array}$ & $\begin{array}{c}\mathrm{U} \\
\text { (percent) }\end{array}$ & $\begin{array}{c}\text { Au } \\
\text { (oz/ton) }\end{array}$ & $\underset{\text { (or/ton) }}{\mathrm{Ag}}$ & $\begin{array}{c}\mathrm{Cu} \\
\text { (percent) }\end{array}$ & $\begin{array}{c}\mathrm{Pb} \\
\text { (percent) }\end{array}$ & $\begin{array}{c}\mathrm{Zn} \\
\text { (percent) }\end{array}$ & \\
\hline 62 & $C-2$ & Nancy & $\begin{array}{l}\text { Crosscut adit, } \\
\text { drifts, stopes }\end{array}$ & 1.200 & .10 & -- & -- & -. & -- & -- & -- & -- & \\
\hline 68 & $A-1$ & Unknown & Drift adit & 150 & 0.06 & -- & -- & -- & - & -- & -. & -- & $\begin{array}{l}\text { Vein with pyrite, chalcopyrite (?), } \\
\text { secondary copper minerals. }\end{array}$ \\
\hline 64 & $C-1$ & do. & do. & 100 & .09 & -- & -. & -- & -. & -- & -. & -- & \\
\hline 65 & $C-1$ & do. & do. & 200 & .05 & -- & -. & -- & -- & -. & -- & -. & \\
\hline f6 & $B-3$ & do. & dn. & 100 & .30 & 0.024 & 0.008 & 0.13 & 1.17 & $<0.01$ & 0.01 & 0.02 & Chip sample of vein material at face. \\
\hline 67 & $A-3$ & do. & do. & 100 & .08 & -- & -- & -- & -- & $\cdots$ & -. & -- & $=$ \\
\hline \multirow[t]{2}{*}{68} & $A-3$ & do. & $\begin{array}{l}\text { Drift adit, } \\
\text { crosscut. drift }\end{array}$ & 300 & .40 & .015 & .002 & Tr. & .60 & $<01$ & $<01$ & .04 & $\begin{array}{l}\text { Sample no, } 68-16 \text {-inch chip-channel of } \\
\text { vein material. }\end{array}$ \\
\hline & & & & & .70 & .19 & .12 & Tr. & $\mathbf{T}$. & $<01$ & $<.01$ & .02 & $\begin{array}{l}\text { Sample no. } 68 \text { a--chip from thin fracture } \\
\text { exposed in crosscut. with pyrite and } \\
\text { sooty pitchblende (?). }\end{array}$ \\
\hline 69 & $A-3$ & $\begin{array}{l}\text { Belle of } \\
\text { Memphis }\end{array}$ & Drift adit & 100 & .15 & -- & -- & $\cdots$ & -- & $-\cdot$ & -- & -- & $\begin{array}{l}\text { Vein with pyrite. sphalerite. and } \\
\text { chalcopyrite (?). }\end{array}$ \\
\hline 70 & $A-3$ & Unknown & $\begin{array}{l}\text { Drift adit. } \\
\text { drift }\end{array}$ & 500 & 1.5 & -- & $\cdots$ & -- & -- & -- & $-\cdot$ & -- & $\begin{array}{l}\text { One specimen from vein near face of } \\
\text { east drift shows "hard" pitchblende. }\end{array}$ \\
\hline 71 & $B-3$ & do. & Drift adit & 400 & .10 & $\cdots$ & $\cdot-\cdot$ & -- & -- & -. & -- & -. & \\
\hline 72 & $\mathbf{B - 3}$ & do. & do. & 100 & .04 & -- & -. & -- & -- & .- & -- & - & \\
\hline 73 & $8-3$ & do. & do. & 200 & .05 & -- & -- & -- & -- & -. & -- & -- & \\
\hline 74 & $B-3$ & do. & do. & 500 & .15 & 0.006 & 0.301 & 0.14 & 1.10 & 0.01 & $<0.01$ & 0.02 & $\begin{array}{l}\text { Bulk sample of abnormally radioactive } \\
\text { vein material. }\end{array}$ \\
\hline
\end{tabular}


Table 1. --Tabulated descriptions of localities examined for radioactivity in the Gold Hill mining area, Boulder County, Colorado--Continued

\begin{tabular}{|c|c|c|c|c|c|c|c|c|c|c|c|c|c|}
\hline \multirow[b]{2}{*}{$\begin{array}{l}\text { Locality } \\
\text { numbef } 1 /\end{array}$} & \multirow[b]{2}{*}{$\begin{array}{l}\text { Coordi- } \\
\text { nates } 1 /\end{array}$} & \multirow[b]{2}{*}{ Name } & \multirow{2}{*}{$\begin{array}{l}\text { Type of } \\
\text { workings } \\
\text { examined }\end{array}$} & \multirow{2}{*}{$\begin{array}{l}\text { Length of } \\
\text { workings } \\
\text { trapersed } \\
\text { (feet) 2/ }\end{array}$} & \multirow{2}{*}{$\begin{array}{l}\text { Maximum } \\
\text { radio- } \\
\text { activity } \\
(\mathrm{mr} / \mathrm{hr})\end{array}$} & \multicolumn{7}{|c|}{ Analytical data } & \multirow[b]{2}{*}{ Remarks $\underline{3} /$} \\
\hline & & & & & & $\begin{array}{l}\mathrm{eU} \\
\text { (percent) }\end{array}$ & $\begin{array}{c}\mathrm{U} \\
\text { (percent) }\end{array}$ & $\begin{array}{c}\text { Au } \\
\text { (oz/ton) }\end{array}$ & $\begin{array}{l}\mathrm{Ag} \\
(\mathrm{oz} / \mathrm{ton})(\mathrm{pe}\end{array}$ & $\begin{array}{l}\mathrm{Cu} \\
\text { ercentl }\end{array}$ & $\begin{array}{c}\mathrm{Pb} \\
\text { (percent) }\end{array}$ & $\begin{array}{c}\mathrm{Zn} \\
\text { (percent) }\end{array}$ & \\
\hline 76 & B-3 & do. & do. & 500 & .08 & $\cdots$ & -- & -- & -- & -- & -- & -. & \\
\hline 77 & $B-3$ & do. & do. & 150 & .08 & - & -- & -- & -- & $\because=$ & -- & -- & \\
\hline 78 & $A-3$ & $\begin{array}{l}\text { Pine Cone } \\
\text { claim }\end{array}$ & do. & 300 & .10 & -- & -- & -- & -- & -- & $\cdots$ & -- & \\
\hline 79 & B-2 & $\begin{array}{l}\text { Unknown } \\
\text { (Black Cloud } \\
\text { vein) }\end{array}$ & do. & 150 & .15 & -- & -- & -- & -- & -- & -- & -- & \\
\hline 80 & B-2 & Unknown & do. & 150 & .15 & -- & -- & -- & $\cdots$ & -- & -- & -- & \\
\hline 81 & B-2 & $\begin{array}{l}\text { Bighorn } \\
\text { tunnel }\end{array}$ & do. & 200 & .06 & -- & -- & -- & -- & -- & - & -- $v$ & Vein with pyrite and chalcopyrite (?). \\
\hline 82 & $B-3$ & Unknown & $\begin{array}{l}\text { Drift adit, } \\
\text { drifts, crosscut } \\
\text { stopes. }\end{array}$ & 1.100 & 0.15 &.- & -- & -- & -- & - & -- & -- & \\
\hline \multirow[t]{2}{*}{89} & D-3 & $\begin{array}{l}\text { Yellow } \\
\text { Pine tunnel }\end{array}$ & do. & 3.000 & .03 & 0.004 & -- & Tr. & 2.40 & 0.03 & $<0.01$ & 0.01 & $\begin{array}{l}\text { Sample no. } 83-- \text { grab sample. from ore } \\
\text { chute near caved on east drift. }\end{array}$ \\
\hline & & & & & .70 & .027 & 0.006 & Insuffi & icient sample & e .01 & $<01$ & .02 & $\begin{array}{l}\text { Sample no, } 83 \text { a-chip from face of drift. } \\
\text { Pegmatite mineral may be source of radioactivity }\end{array}$ \\
\hline \multirow[t]{4}{*}{84} & $B-2$ & $\begin{array}{l}\text { Unknown } \\
\text { (Goldsmith } \\
\text { Maid vein) }\end{array}$ & $\begin{array}{l}\text { Drift adit } \\
\text { winze. drift }\end{array}$ & 40 & 2.00 & .40 & .076 & $\mathbf{T r}$ & 3.22 & .02 & .33 & .18 & $\begin{array}{l}\text { Sample no. 84--chip of vein material showing } \\
\text { sooty pitchblende. }\end{array}$ \\
\hline & & & & & 2.00 & .064 & .018 & .06 & 12.56 & .06 & .71 & .85 & $\begin{array}{l}\text { Sample no. } 84 a--6-\text { inch chip-channel of } \\
\text { vein at radioactive locality. }\end{array}$ \\
\hline & & & & & 2.00 & .20 & .095 & Tr. & .14 & .16 & $<01$ & $<01$ & $\begin{array}{l}\text { Sample no. 84-1-chip of radioactive vein } \\
\text { material showing sooty pitchblende. }\end{array}$ \\
\hline & & & & & 2.00 & .69 & .30 & Tr. & 10.40 & .21 & 3.05 & .41 & $\begin{array}{l}\text { Sample no. } 84-2-\text {-chip of radioactive vein } \\
\text { material showing sooty pitchblende. } \\
\text { torbernite, and metatorbernite. }\end{array}$ \\
\hline
\end{tabular}


Table 1. - Tabulated descriptions of localities examined for radioactivity in the Gold Hill mining area, Boulder County, Colorado--Continued

\begin{tabular}{|c|c|c|c|c|c|c|c|c|c|c|c|c|c|}
\hline \multirow[b]{2}{*}{$\begin{array}{l}\text { Locality } \\
\text { number } 1 /\end{array}$} & \multirow[b]{2}{*}{$\begin{array}{l}\text { Coordi- } \\
\text { nates } \underline{1} \text { ' }\end{array}$} & \multirow[b]{2}{*}{ Name } & \multirow{2}{*}{$\begin{array}{l}\text { Type of } \\
\text { workings } \\
\text { examined }\end{array}$} & \multirow{2}{*}{$\begin{array}{l}\text { Length of } \\
\text { workings } \\
\text { traversed } \\
\text { (feet) } \underline{2}\end{array}$} & \multirow{2}{*}{$\begin{array}{l}\text { Maximum } \\
\text { radio- } \\
\text { activity } \\
\text { (mr/hr) }\end{array}$} & \multicolumn{7}{|c|}{ Analytical data } & \multirow[b]{2}{*}{ Remarks 3 / } \\
\hline & & & & & & $\begin{array}{l}\mathrm{eU} \\
\text { (percent) }\end{array}$ & $\begin{array}{c}\mathrm{U} \\
\text { percent) }\end{array}$ & $\begin{array}{c}\text { Au } \\
\text { (oz/ton) }\end{array}$ & $\frac{\mathrm{Ag}}{(02 / \text { ton })}$ & $\begin{array}{c}\mathrm{Cu} \\
\text { (percent) ( }\end{array}$ & $\begin{array}{c}\mathrm{Pb} \\
\text { (percent) }\end{array}$ & $\frac{\mathrm{Zn}}{\text { (percent) }}$ & \\
\hline 85 & $B-2$ & $\begin{array}{l}\text { Unknown } \\
\text { (Hoosier } \\
\text { reef) }\end{array}$ & $\begin{array}{l}\text { Drift adit, } \\
\text { drifts on } \\
\text { several veins }\end{array}$ & 700 & 0.30 & - & -- & -- & -- & $\cdots$ & -- & $-\cdot$ & \\
\hline 86 & $B-2$ & $\begin{array}{l}\text { Goldsmith } \\
\text { Maid }\end{array}$ & $\begin{array}{l}\text { Crosscut adit, } \\
\text { drifts }\end{array}$ & 200 & .05 & -- & -- & -- & -- & -- & -- & -- & \\
\hline 87 & $B-2$ & Unknown & $\begin{array}{l}\text { Crosscut adit. } \\
\text { drifts, stopes }\end{array}$ & 800 & .70 & 0.32 & 0.31 & Tr. & 0.10 & $<0.01$ & $<0.01$ & 0.04 & $\begin{array}{l}\text { Chip of radioactive vein material with } \\
\text { sooty pitchblende (?) and schroeckingerite (?). } \\
\text { at intersection of } 2 \text { veins. }\end{array}$ \\
\hline
\end{tabular}




\section{Radioactive depastriat tocal1ty 68}

Three veins are exposed by the workings at locality 68 (fig. 3). The adit follows a vein composed dominantly of horn quartz; it strikes nearly north and dips $54^{\circ}$ to $56^{\circ}$ east. A crosscut to the west and southwest exposes a very narrow, apparently discontinuous, vertical vein that strikes nearly east and contains pyrite and sooty pitchblende (?). At its southwestern end the crosscut intersects a vein that strikes northwesterly and dips $36^{\circ}$ to $48^{\circ}$ northeast; this vein has been exposed by about 100 feet of drift. The predominant country rock is granite.

The east-trending vein is the most radioactive of the three; the average radioactivity over the length of its exposure is $0.30 \mathrm{mr} / \mathrm{hr}$, and the maximum is $0.70 \mathrm{mr} / \mathrm{hr}$ at the place where sample $68 \mathrm{a}$ (table 1) was taken. The northwest-trending vein has an average radioactivity of $0.20 \mathrm{mr} / \mathrm{hr} \mathrm{and}$ several local "highs" of 0.40 to $0.50 \mathrm{mr} / \mathrm{hr}$ on a few fracture surfaces. The north-trending vein has an average radioactivity of $0.70 \mathrm{mr} / \mathrm{hr}$ with a few local maxima of 0.10 to $0.15 \mathrm{mr} / \mathrm{hr}$. The average radioactivity of the barren country rock exposed in the crosscut is $0.04 \mathrm{mr} / \mathrm{hr}$.

The north-trending vein and the northwest-trending vein are superficially similar in composition, although the northwest vein is much more radioactive. The vein material is predominantly horn quartz as stringers, lenticular bodies, and cementing fragments of fractured and altered wall rock, Limonite and clay coat fracture surfaces, and gouge is commonly present along fractures in the veins. Megascopically visible metallic mineral grains are very sparse in both of these veins, but close examination of hand specimens from the northwest-trending vein shows fine-grained pyrite (possibly with some chalcopyrite) and minute flecks of a gray-black mineral ( possibly a telluride) disseminated in the horn quartz. Both veins appear continuous and are more than a foot thick.

The thin east-trending vein exposed in the crosscut contains finely disseminated pyrite and a black mineral tentatively identified as sooty pitchblende. This vein ranges in thickness from a fraction of an inch to about three inches over the length of its exposure. It is apparently discontinuous as no intersections with the other two veins were observed. 


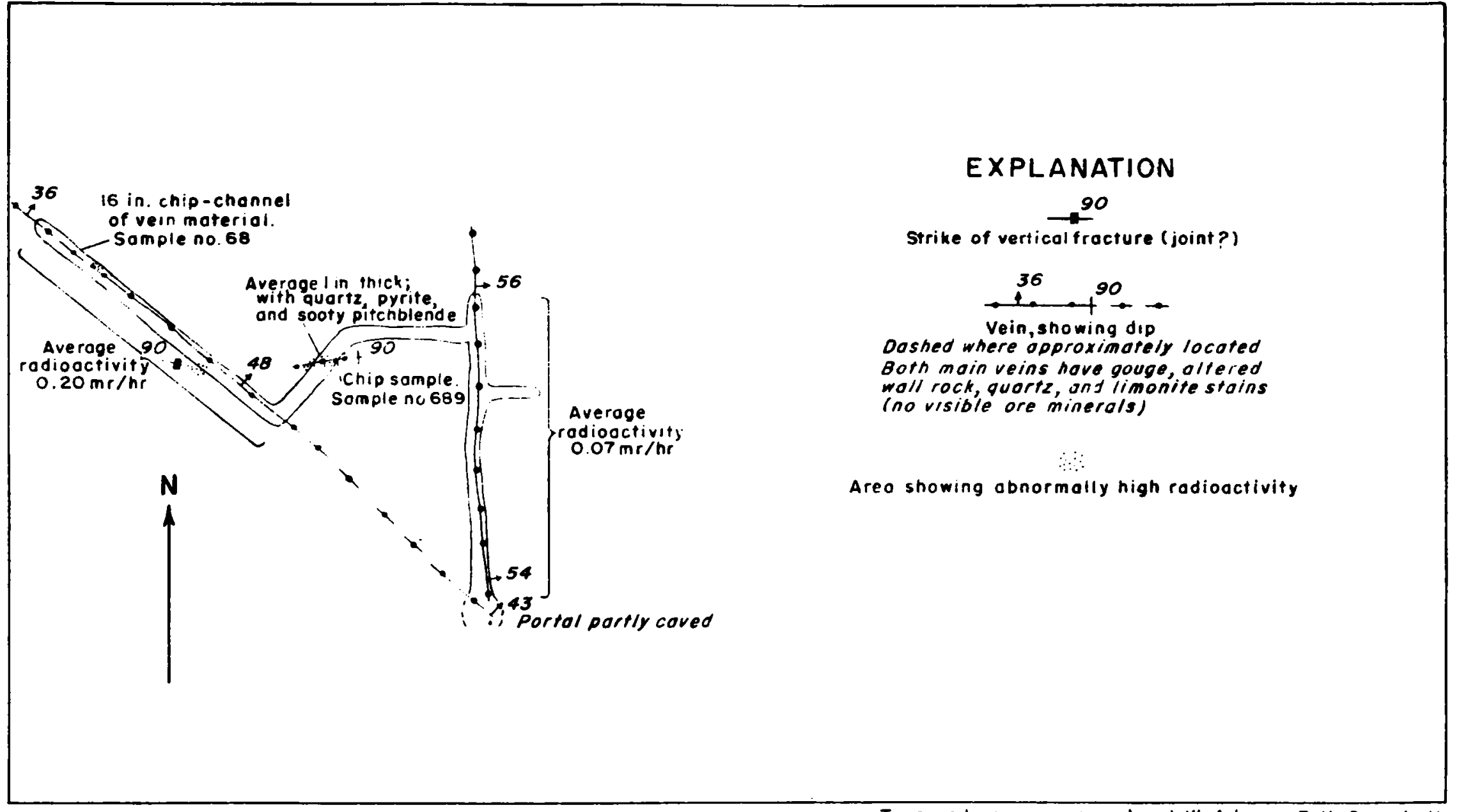

Tape and compass map by J.W. Adams, R. H. Cainpbell,

FIGURE 3 .- GEOLOGIC SKETCH OF WORKINGS AT LOCALITY 68,

GOLD HILL MINING AREA, SHIOWING PLACES SAMPLED

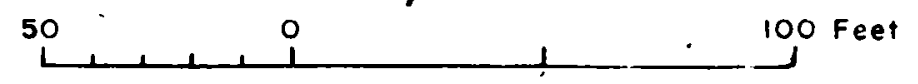


Radioactive deposit at locality 84

The Goldsmith Maid vein is exposed in the prospect at locality 84 . It strikes N. $50^{\circ} \mathrm{E}$, at the face of the adit and dips generally $73^{\circ} \mathrm{NW}$. although both walls have local irregularities of dip. The vein is about 5 feet thick at the face. It is composed chiefly of lenses and interlacing stringers of gray horn quartz (locally with some milky quartz and clear quartz) which also cements abundant fragments of argillized, chloritized, and in places pyritized granite. Pyrite, galena, and sphalerite occur in small pods and thin seams in both the horn quartz and the fragments of altered wall rock. Fine-grained pyrite and minute flecks of an unidentified gray-black mineral (possibly a telluride) are commonly disseminated in the horn quartz; in places they are more concentrated adjacent to wall rock fragments and locally occur as nearly complete rims on fragments.

The country rock is chiefly Boulder Creek granite which, adjacent to the vein. is commonly sheared, argillized,chloritized, and locally pyritized. The alteration extends several feet into the hanging wall; it also occurs in the footwall where poor exposures prevent any reasonable estimate of its extent.

The exposed extent of the radioactive deposit is shown on figure 4, and analyses of samples are given in table 1. As the deposit extends for an unknown distance into the altered wall rock on the northwest side of the vein, its true thickness cannot be detelmined.

Uranium minerals coat fractures and small shears in the vein and the altered Boulder Creek granite on the hanging-wall side (northwest side) of the vein and are disseminated in the sheared and altered country rock in the hanging wall. A sooty black mineral tentatively identified as pitchblende, is the source of much of the radioactivity. It is however, very similar in appearance to a non-radioactive mineral (probably a manganese oxide) that is abundant. Torbernite was identified by $\mathrm{X}$-ray methods by W. F. Outerbridge, U. S. Geological Survey, Denver Laboratory. A fluorescent mineral intimately associated with the torbernite and identical with it in megascopic appearance is believed to be metatorbernite. It is possible that all of these uranium minerals are of secondary origin and were emplaced by migrating ground water; therefore, no genetic relation could be established between the uranium minerals and the primary vein material. 


$$
\text { N }
$$

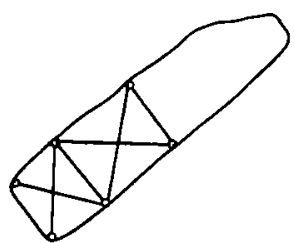

Plan of sub-level

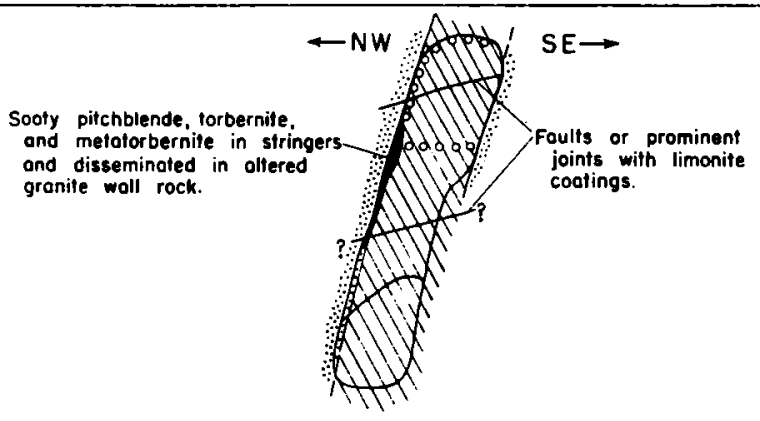

Cross section at foce of odit level showing vertical extent of rodiooctive deposit (solid black)
Woll rock is sheored, orgillized. pyritized Boulder

Creek granite with locol stringers of horn quartz,

in ploces corrying sulfides.

High rodiooctivity is restricted to left rib.

(Hanging wall side)

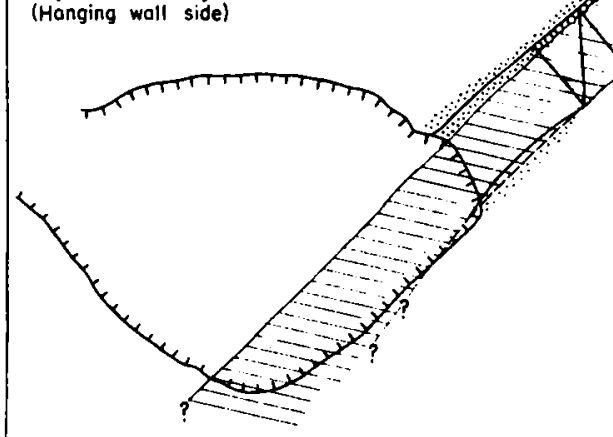

Geologic mop of adit and open cut

Vein is breccioted, oltered Boulder Creek granite ond pegmotite with obundont horn quortz cement; locol stringers and goleno spholerife, pyrite. ond possible rellurides.

Uranium minerols appear to be secondory.
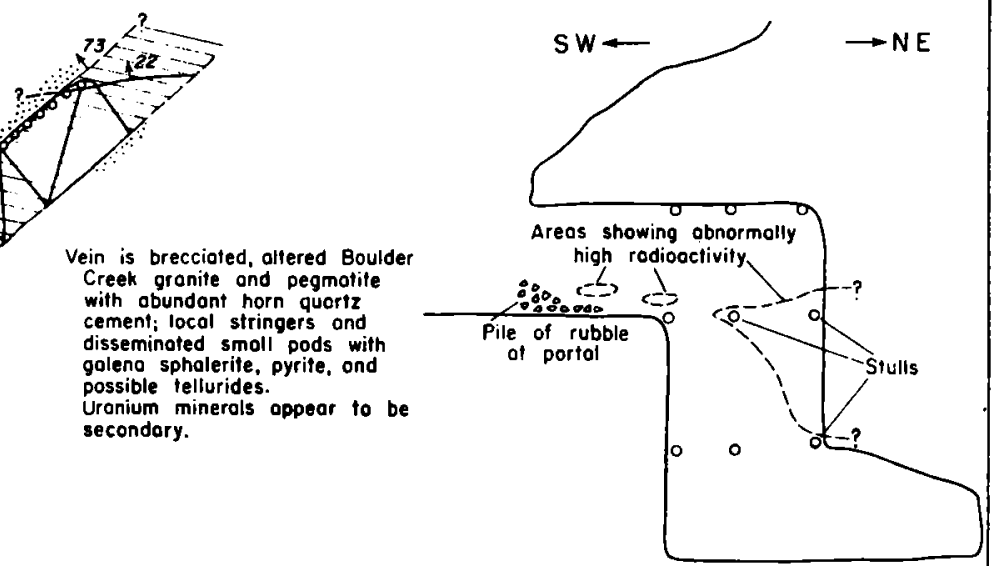

Vertical longitudinal projection along honging woll of vein

\section{EXPLANATION}

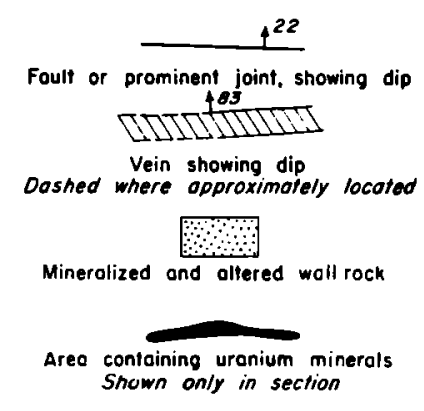

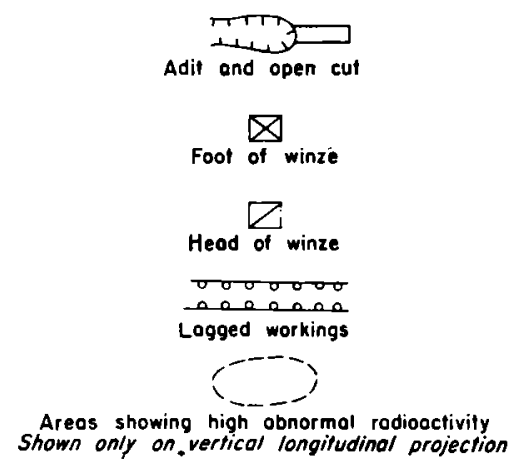

Tope and composs mop by $R$. $H$ Compbell $E$. Roseboom, Jr., ond R. U. King, August 1953

FIGURE 4-GEOLOGIC MAP AND SECTIONS OF WORKINGS AT LOCALITY 84, GOLD HILL MINING AREA, SHOWING KNOWN EXTENT OF RADIOACTIVE DEPOSIT. 
Radioactive deposit at locality 70

Pitchblende was found in a sample of highly radioactive vein material about 25 feet from the face of the easternmost drift in the workings at locality number 70. One piece of vein material about 5 inches in diameter contained all of the highly radioactive material; it was entirely removed in sampling. The average radioactivity of the vein is $0.08 \mathrm{mr} / \mathrm{hr}$ and the maximum radioactivity (material sampled) was $1.50 \mathrm{mr} / \mathrm{hr}$.

The vein material sampled consists primarily of brecciated granitic rock, well cemented by intersecting stringers and irregular fillings of horn quartz. Pyrite, galena, and pitchblende were observed in polished sections cut from the sample. Pyrite is abundant as isolated grains disseminated throughout the vein quartz, and also occurs as patches and locally as veinlets in both the breccia fragments and the vein quartz. Galena is much less abundant than pyrite. It occurs as highly irregular patches and locally as small veinlets in both breccia fragments and vein quartz. Pitchblende is approximately of the same abundance as galena. Very thin veinlets of pitchblende partly rim fragments of brecciated wall rock and locally penetrate the fragments along minute irregular cracks. Pitchblende also occurs in small patches in the breccia fragments and locally forms irregular patches and veinlets in the vein quartz. Some of the veinlets have borders with shapes that suggest a colloform structure for the pitchblende. The paragenetic sequence pyrite-pitchblende-galena-pyrite is suggested from studies of the polished sections; however, the limited data available are insufficient to establish the relations of these ore minerals with any degree of certainty and the proposed sequence is highly tentative. 


\section{RADIOACTIVITY AND SULFIDE ZONING}

There is some suggestion that sulfide zoning might be an effective prospecting guide to radioactive deposits in the Gold Hill mining area. Although the data on areal distribution are limited and the significance of the pattern shown on figure 2 is questionable, the distribution of the sulfide ores as compiled by McReel suggests a possible zoning. Pyritic gold ores (locally with abundant free gold) predominate in two small and poorly defined centers: the area of the $\mathrm{T} w$ in and Klondike veins in the northwest part of the district, and the area of the Grand Republic mine in the southeast. These centers are partly surrounded by veins containing lead-silver ores. The telluride ores appear to be evenly distributed throughout the district and show no apparent relation to the zoning of the sulfide ores. A belt, containing telluride veins but apparently lacking important sulfide deposits, trends northeast through the area between the northwest and southeast zones containing lead-silver ores.

On the basis of the apparent zonal relations of uranium deposits in other districts showing a zonal distribution of sulfide-bearing veins (for example: Central City, Colo.. Leonard, 1952), it was inferred that the most favorable zonal position fór uranium deposits in the Gold Hill area would be in those areas containing both pyritic gold and lead-silver ores. These favorable zones, shown as stippled areas on figure 2, are roughly intermediate in zonal position between the pyritic gold centers and the zones containing lead-silver ores. The favorable zone related to the northwestern pyritic center is the larger and the better developed of the two, although both are indistinct and the limits shown on figure 2 are at best only approximate. Additional data on the mineralogy of the individual deposits might alter considerably the size and shape of these zones, or possibly even disprove the existence of any zonal pattern in this district. As most of the veins examined showed no megascopically visible ore minerals, little was done during the course of this examination to refine the zonal pattern as compiled from published sources prior to beginning the field work. 


\section{CONCLUSIONS}

Nearly all the veins examined, regardless of zonal position, are noticeably more radioactive than the adjacent country rocks. If these veins constitute a representative sample, radioactivity slightly higher than that commonly found in the country rocks is typical of the veins of the area. This suggests that some radioactive material was probably introduced with nearly all of the veinfilling material. Although the significance of this widespread higher radioactivity is difficult to elvaluate with the present data, a more detalledstudy of this feature might lead to a radioactivity method useful in prospecting for base - and precious-metal veins at least in the Gold Hill area.

The known uranium deposits in the Gold Hill area are small and fairly widely scattered and are probably not of immediate economic importance. Most of the deposits found during this reconnaissance. having a grade of 0.10 percent $\mathrm{U}$ or more, are estimated to contain less than 1 ton of uranium ore. If the size and grade of the known deposits is considered indicative of the size and grade of any undiscovered deposits, it is inferred that very few, if any, are of sufficient size and grade to be worked economically for uranium alone. If, however, the district should again become active in the mining of gold and silver, or possibly, lead and/or zinc, some uranium might be recovered as 3 byproduct.

All of the deposits where uranium minerals have been observed are within the northwestern favorable zone (fig. 2). In nine radioactive deposits where uranium minerals were not observed samples show 0.10 percent $\mathrm{eU}$ or more; six of these are in the northwestern favorable zone. Thus, although radioactive deposits are not restricted to this favorable zone, it is possible that the concept of a preferred zonal position for uranium is applicable to the Gold Hill mining area. The writer, however, recommends caution in the application of this zonal pattern in prospecting because it is poorly defined by the present data and because a genetic relation between uranium and the sulfide ores has not been definitely established. 
As ore minerals were observed in only a very few of the veins examined, little can be concluded as to whether the uranium deposits are genetically associated with the telluride ores or the sulfide. ores. An examination of the map (fig. 2). however, shows that most of the deposits containing 0.10 percent or more $\mathrm{eU}$ are in the set of veins bearing east-northeast. As these veins also contain the sulfide ores (Goddard, 1940), some relationship between the uranium deposits and the sulfide ores is suggested. However, more data are needed concerning the relationship of the uranium deposits with the other ores to determine more precisely if the uranium is genetically related to the sulfide ores and involved in their zoning. If the uranium is related to the telluride ores which do not appear to be affected by the zoning of the sulfides, a knowledge of the zoning may be expected to be less effective, or even useless, in prospecting this district for_newic uranium deposits.

Within the northwestern favorable zone there are two areas (one near Butzel Mountain and another in the vicinity of Summerville) where uraniferous deposits appear to be grouped. These groups of deposits offer opportunities to study the occurrence of uranium minerals within their geologic setting. The large number of accessible underground workings would be especially helpful in such studies.

\section{LITERA T URE CITED}

Goddard, E. N. , 1940, Preliminary report on the Gold Hill mining district, Boulder County, Colorado: Colorado Sci. Soc. Proc., v. 14, no. 4. p. 103-139.

Leonard, B. F.. 1952, Relation of pitchblende deposits to hypogene zoning in the Front Range mineral belt, Colorado: Econ. Geology, v. 47, no. 7, p. 773.

Lovering, T. S., 1932, Relations of ore deposits to geologic structure in Boulder County, Colorado: Colorado Sci. Soc. Proc.. v. 13, no. 3, p. 77-88.

Lovering. T. S., and Goddard, E. N.. 1950, Geology and ore deposits of the Front Range, Colorado: U. S. Geol. Survey Prof. Paper 223, p. 234-255.

\section{UNPUBLISHED REPORT}

King, R. U., in preparation. Reconnaissance investigations for uranium in the Colorado Front Range and adjacent areas, 1947-1951: U. S. Geol. Survey Trace Elements Inv. Rept. 59. 



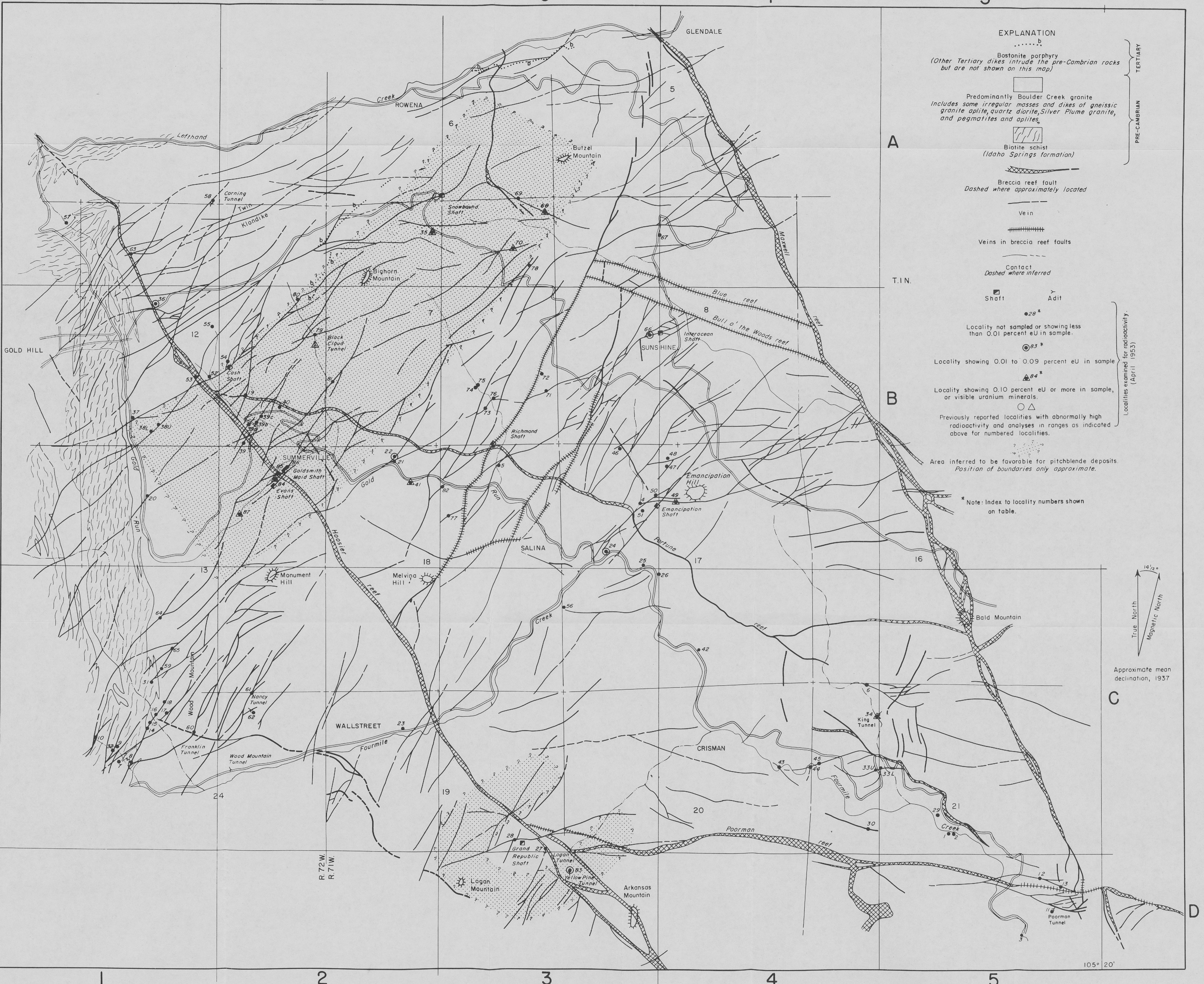

B

1 